\title{
Intraluminal carotid thrombosis and acute ischemic stroke associated with COVID-19
}

\author{
Diaa Hamouda $^{1} \cdot$ Dinesh V. Jillella $^{1} \mathbb{C} \cdot$ Nirav Bhatt $^{1} \cdot$ Sitara Koneru $^{1} \cdot$ Michael R. Frankel $^{1} \cdot$ Raul G. Nogueira $^{1}$
}

Received: 2 February 2021 / Revised: 9 April 2021 / Accepted: 12 April 2021 / Published online: 29 April 2021

(c) Springer-Verlag GmbH Germany, part of Springer Nature 2021

\begin{abstract}
COVID-19 (Coronavirus disease 2019) caused by SARS-CoV-2 has a diverse constellation of neurological manifestations that include encephalopathy, stroke, Guillain-Barré syndrome, myelitis, and encephalitis. Intraluminal carotid thrombi (ILT) are infrequent lesions seen in only $1.6 \%$ of patients with acute ischemic stroke. Underlying atherosclerosis is the most common lesion associated with ILT formation. However, with COVID-19, we have encountered ILT in patients without significant atherosclerotic disease. The endothelial inflammation and hypercoagulable state associated with COVID-19 pose a risk of arterial and venous thromboembolism and could have contributed to this presentation although the exact pathophysiology and optimal treatment of ILT in COVID-19 remain elusive. Herein, we present a series of ischemic stroke patients with carotid ILT in the setting of a recent SARS-CoV-2 infection.
\end{abstract}

Keywords Acute stroke $\cdot$ Carotid thrombus $\cdot$ Intraluminal thrombus $\cdot$ Ischemic stroke $\cdot$ COVID-19

\section{Introduction}

COVID-19 (Coronavirus disease 2019) caused by SARS$\mathrm{CoV}-2$ has a diverse constellation of neurological manifestations that include encephalopathy, ischemic and hemorrhagic strokes, Guillain-Barré syndrome, myelitis and encephalitis. Intraluminal carotid thrombi (ILT) are infrequent lesions seen in only $1.6 \%$ of patients with acute ischemic stroke (AIS) [1]. Underlying atherosclerosis is the most common lesion associated with ILT formation and up to $80 \%$ of carotid ILT cases continue to have $>50 \%$ stenosis after resolution of ILT [1]. However, with COVID-19, we have encountered ILT in patients without significant atherosclerotic carotid artery disease. The endothelial inflammation and hypercoagulable state associated with COVID-19 pose a risk of arterial and venous thromboembolism [2-4]. Furthermore, there are several reports of patients with systemic thromboembolism despite being on anticoagulation. The exact pathophysiology and optimal treatment remain

Dinesh V. Jillella

dineshvinayakjillella@gmail.com

1 Department of Neurology, Emory University School of Medicine and Marcus Stroke and Neuroscience Center, Grady Memorial Hospital, Atlanta, GA, USA elusive. Herein, we present a series of stroke patients with carotid ILT in the setting of a recent SARS-CoV-2 infection.

\section{Methods and results}

From July 9th, 2020 to August 17th, 2020, 4 patients were identified with AIS with varying thrombus burden but similar morphological patterns of ILT. IRB approval was waived per institutional policies for this retrospective case series of $\leq 5$ patients. The demographic, clinical, radiological, and laboratory characteristics are illustrated in Table 1 and images in Fig. 1. Three patients (patients A, B, C) presented to the hospital emergency department with stroke symptoms. One patient (patient D) was admitted with COVID-19 manifestations who was initially on the hospital floor unit but developed new stroke-like symptoms that were noticed upon awakening on hospital day 5 and was subsequently transferred to the Intensive Care Unit (ICU) due to worsening respiratory status. Patients A and C had mild COVID-19 respiratory symptoms, but patient $\mathrm{A}$ required neurological ICU care for a large stroke management. Patients B and D required medical ICU for COVID-19-related respiratory failure, and notably, had been receiving prophylaxis with therapeutic heparin as protocolled at our institution at the time of the stroke symptom onset. 


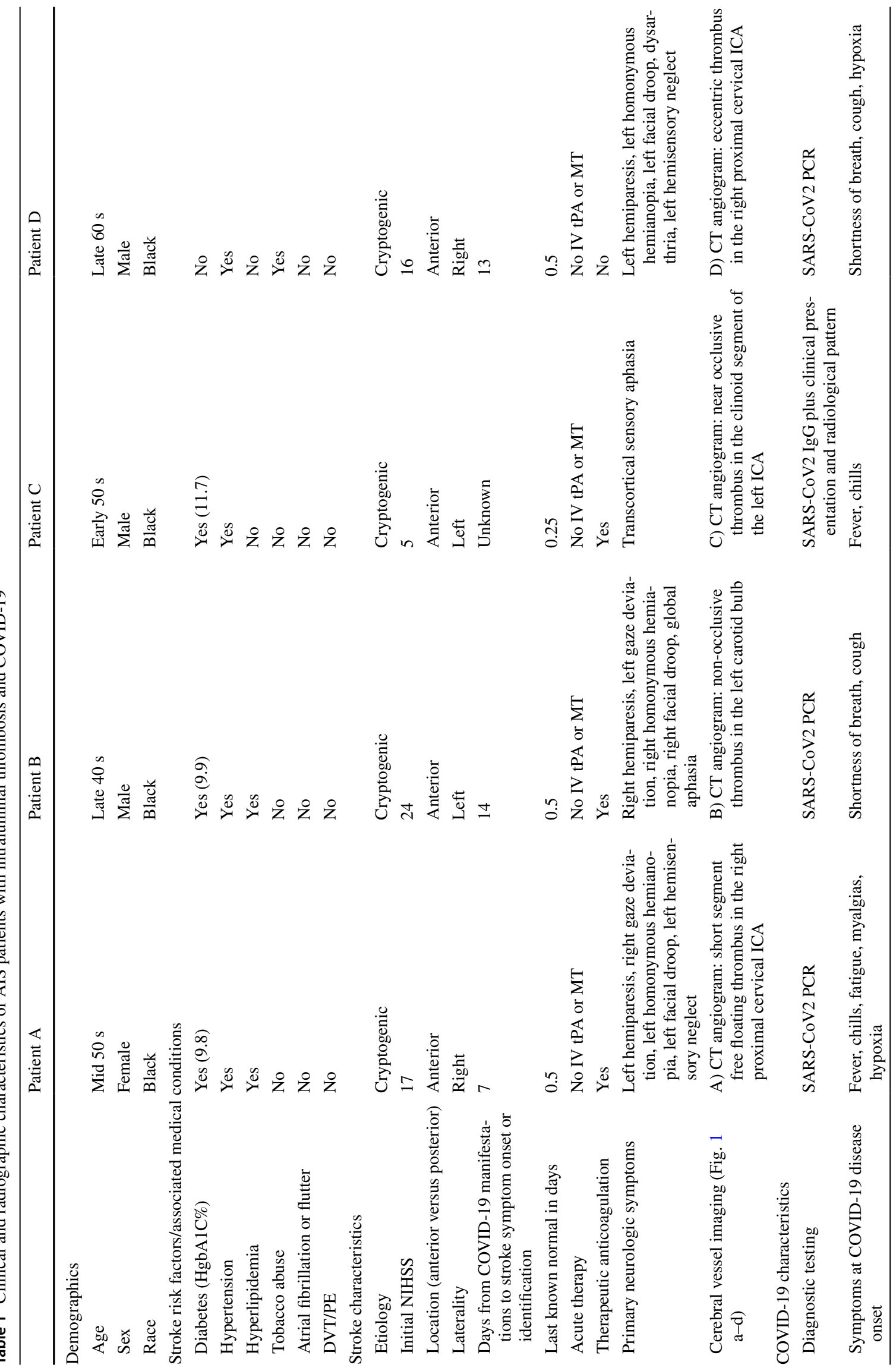




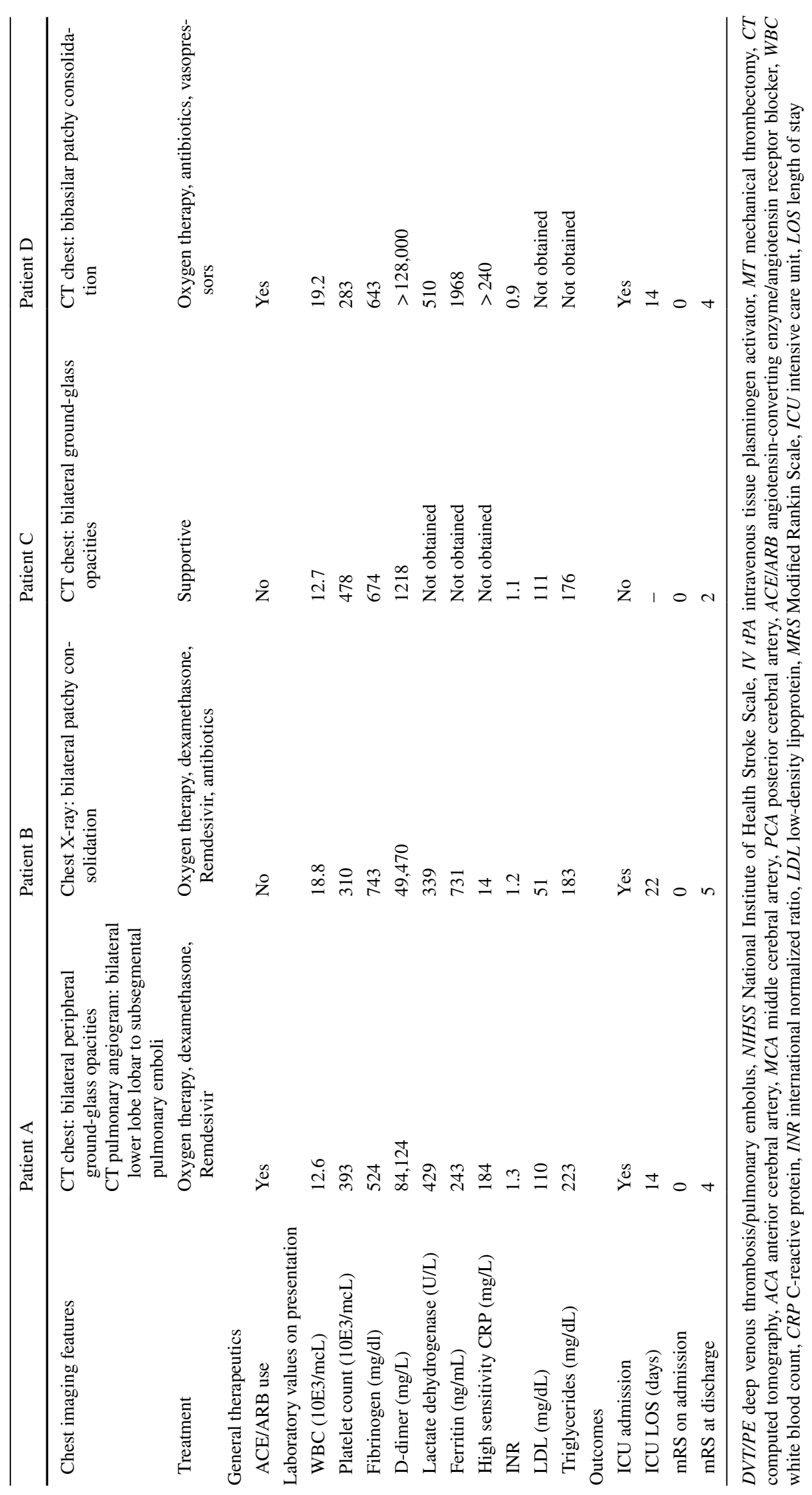



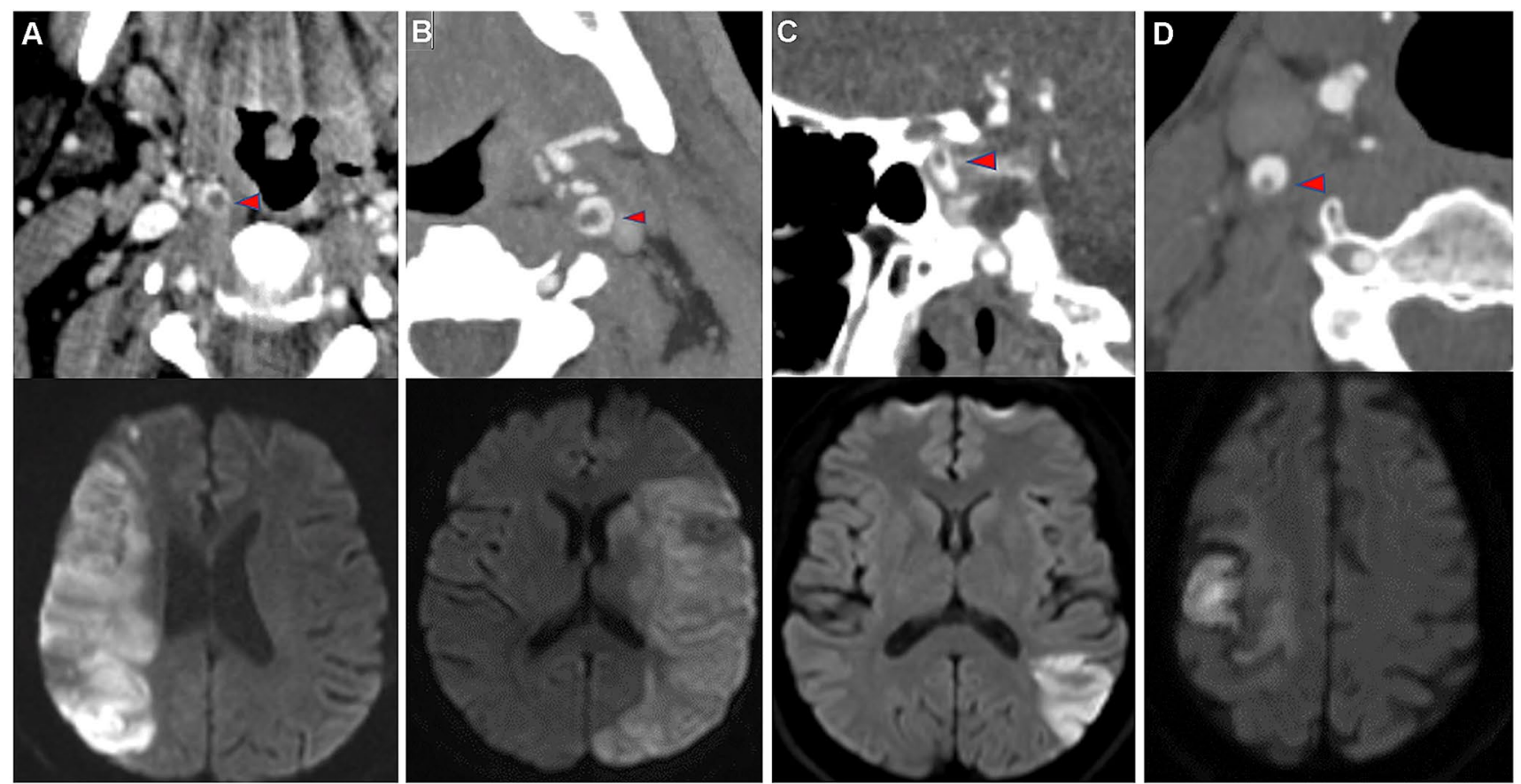

Patient A, CT angiogram of the neck axial view showing intraluminal thrombus in the right cervical ICA (arrow head) on the top and MRI DWI sequence showing restricted diffusion of right MCA territory on the bottom Patient B, CT angiogram of the neck axial view showing intraluminal thrombus in the left cervical ICA (arrow head) on the top and MRI DWI sequence showing restricted diffusion on left MCA territory on the bottom Patient D, CT angiogram of the head axial view showing intraluminal thrombus in the right cervical ICA (arrow head) on the top and MRI DWI sequence showing restricted diffusion on the right MCA territory on the botton

Fig. 1 Radiological characteristics of intraluminal thrombosis in COVID-19 patients

All patients had evidence of recent SARS-CoV-2 infection as confirmed by PCR $(n=3)$ or $\operatorname{IgG}$ antibody $(n=1)$ in addition to clinical and radiological manifestations consistent with COVID-19. Mean age was $55.3 \pm 7.7$ years. Most patients $(n=3)$ had at least two vascular risk factors, and none had a central embolic source, such as cardiac or aortic arch pathology, identified on work-up. D-Dimer was profoundly elevated in 3 patients. ILT involved the cervical $(n=3)$ or intracranial $(n=1)$ segments of the internal carotid artery. Besides ILT, there was no other local vascular pathology identified. COVID-19 symptoms ranged from mild transient respiratory symptoms to severe pneumonia requiring critical care support. None of the patients received acute reperfusion therapy either due to presentation beyond the therapeutic window or due to the absence of favorable vascular or perfusion characteristics on initial imaging.

\section{Discussion}

We describe 4 cases of carotid ILT in nasopharyngeal PCRbased or serologically confirmed COVID-19 patients. The site of ILT in our cohort included cervical ICA at and distal to the bifurcation $(n=3)$ and clinoid ICA $(n=1)$. All patients $(n=4)$ had at least one cardiovascular risk factor and three of them had two or more cardiovascular risk factors. In contrast to prior reports which had a strong correlation of ILT with carotid atherosclerotic disease and stenosis [1], in our cohort, only one patient $(n=1)$ had evidence of ICA mild atherosclerotic stenosis, whereas three patients $(n=3)$ did not have radiologically significant atherosclerotic disease. Even in the recent descriptions of COVID-19 relation to ILT, ILT has been associated with underlying atherosclerotic disease. Two patients described in a paper by D'Anna et al. had a floating thrombus in either the common carotid artery or ICA, and with both these patients having atherosclerosis/plaque associated with the ILT [5]. Similarly, in another series of 6 patients by Mohamud et al., 5 were identified to have a clear atherosclerotic plaque underlying the ILT [6]. Our observation suggests a different pathophysiological pathway to carotid ILT formation in COVID-19 patients with only one patient having mild atherosclerotic disease. Derangement of thrombotic factors, including factor VIII, fibrinogen, D-dimer, and CRP have been consistently observed in COVID-19 patients [2,3]. However, these abnormalities were typically reported in severely affected patients who required intensive care support.

Systemic inflammation plays a vital role in the propagation and destabilization of atherosclerotic plaques [7, 8]. It has been hypothesized that severe inflammatory responses and cytokine release can lead to atherosclerotic plaque destabilization and rupture as was described in the prior study 
that reported ILT in COVID-19 [6]. Additionally, it has also been established that endothelial cells and arterial smooth muscle cells express Angiotensin-converting enzyme-2 (ACE-2) which is the receptor for viral mRNA entry into cells $[4,9]$. In a recent study, viral inclusion bodies were detected in endothelial cells [4]. The pathophysiological consequences of endothelial infection by viral mRNA are yet to be defined, but endothelial injury can promote platelet adhesion and thrombus formation that could have contributed to the occurrence of ILT in our series without obvious underlying atherosclerosis. Although it is difficult to draw definitive conclusions considering the inherent limitations of our observational study, the similar morphological appearance of ILT in COVID-19 patients suggests a distinctive underlying pathophysiology that is not fully defined. It is crucial to identify the underlying process to tailor appropriate managements.

\section{Conclusion}

In this small case series of relatively young patients with acute ischemic stroke, we found an association between COVID-19 infection and ILT. This suggests a distinctive pathophysiological pattern of stroke in COVID-19 patients. Further studies are necessary to elucidate the underlying pathophysiology and target specific therapy.

Funding None.

\section{Declarations}

Conflicts of interest RGN reports consulting fees for advisory roles with Stryker neurovascular, cerenovus, medtronic, phenox, anaconda, genentech, biogen, prolong pharmaceuticals, imperative care and stock options for advisory roles with brainomix, Viz-AI, corindus vascular robotics, Vesalio, ceretrieve, astrocyte and cerebrotech. DCH is a consultant for Stryker and Vesalio and holds stock options at Viz.AI. The other authors have no conflict of interest.

\section{References}

1. Singh R-J, Chakraborty D, Dey S et al (2019) Intraluminal thrombi in the cervico-cephalic arteries. Stroke 50(2):357-364. https://doi.org/10.1161/strokeaha.118.023015

2. Panigada M, Bottino N, Tagliabue P et al (2020) Hypercoagulability of COVID-19 patients in intensive care unit: a report of thromboelastography findings and other parameters of hemostasis. J Thromb Haemost 18(7):1738-1742. https://doi.org/10.1111/jth. 14850

3. Spiezia L, Boscolo A, Poletto F et al (2020) COVID-19-related severe hypercoagulability in patients admitted to intensive care unit for acute respiratory failure. Thromb Haemost 120(06):9981000. https://doi.org/10.1055/s-0040-1710018

4. Varga Z, Flammer AJ, Steiger P et al (2020) Endothelial cell infection and endotheliitis in COVID-19. Lancet 395(10234):14171418. https://doi.org/10.1016/s0140-6736(20)30937-5

5. D’Anna L, Kwan J, Brown Z et al (2020) Characteristics and clinical course of COVID-19 patients admitted with acute stroke. J Neurol 267(11):3161-3165. https://doi.org/10.1007/ s00415-020-10012-4

6. Mohamud AY, Griffith B, Rehman M et al (2020) Intraluminal carotid artery thrombus in COVID-19: another danger of cytokine storm? Am J Neuroradiol 41(9):1677-1682. https://doi.org/10. 3174/ajnr.A6674

7. Libby P (2002) Inflammation in atherosclerosis. Nature 420(6917):868-874. https://doi.org/10.1038/nature01323

8. Vanderlaan PA, Reardon CA, Getz GS (2004) Site specificity of atherosclerosis. Arterioscler Thromb Vasc Biol 24(1):12-22. https://doi.org/10.1161/01.atv.0000105054.43931.f0

9. Ferrario CM, Jessup J, Chappell MC et al (2005) Effect of angiotensin-converting enzyme inhibition and angiotensin ii receptor blockers on cardiac angiotensin-converting enzyme 2. Circulation 111(20):2605-2610. https://doi.org/10.1161/circulationaha.104. $510461 . \mathrm{i}$ 